\title{
Severe Acute Respiratory Syndrome (SARS)-Coronavirus (CoV)-2 Versus SARS-CoV: So Similar Yet So Different
}

\section{Saloni Gupta* and Sonam Gupta}

Independent Scholar, Delhi, India

*Corresponding Author: Saloni Gupta, Independent Scholar, Delhi, India.
Received: August 11, 2020;

Published: September 16, 2020

(C) All rights are reserved by Saloni Gupta and Sonam Gupta.

\section{Abstract}

Starting in the Wuhan city of China, the indiscriminate spread of Severe acute respiratory syndrome coronavirus-2 (SARS-CoV-2) and sudden escalation in the number of Coronavirus disease-2019 (COVID-19) cases has brought desolation to mankind. To control the ongoing disastrous pandemic, it is important to congregate the highest level of information on the ecology of this notorious microbe. The similarities between SARS-CoV-2 and SARS-CoV can be traced back to their phylogenetic relationship, however, gaining insight into the divergent features of the novel coronavirus demands extensive research. This article brings together the previously known facts about SARS-coronaviruses and contrasts them with the new findings on SARS-CoV-2 from the most recent studies for future reference.

Keywords: SARS-CoV-2; SARS-CoV; COVID-19; SARS; Coronaviruses; ACE2

\section{Introduction}

Severe acute respiratory syndrome coronavirus-2 (SARSCoV-2), infamously responsible for causing one of the largest pandemics of $21^{\text {st }}$ century, the Coronavirus disease-2019 (COVID-19) outbreak (2019 - 2020), has emerged unpredictably creating a state of global health emergency. By the end of June 2020, the WHO COVID-19 dashboard recorded around 10 million confirmed cases and deaths exceeding 5 lakhs worldwide. SARS coronavirus (SARS-CoV or SARS-CoV-1), the ancestor to SARS-CoV-2, was the culprit behind the 2003 Severe acute respiratory syndrome (SARS) outbreak (2002 - 2004). The SARS outbreak gave rise to more than 8000 reported cases globally with a mortality rate of $\sim 10 \%$.

Coronaviruses are a group of related RNA viruses that infect and cause diseases in mammals and birds. They typically produce respiratory as well as gastrointestinal symptoms among different species they infect. In humans, these viruses cause clinical manifestations ranging from mild upper respiratory tract infection to a lethal form of pneumonia, the Severe acute respiratory syndrome (SARS).

This article comprehensively discusses the biology of SARScoronaviruses, SARS-CoV-2 and SARS-CoV, with emphasis on the unique properties of SARS-CoV-2.

\section{Evolution and zoonosis}

The novel coronavirus, SARS-CoV-2, is homologous to SARS-CoV and shares about $80 \%$ of the gene sequence of SARS-CoV. While the six major open reading frames (ORFs) of SARS-CoV-2 genes share less than $80 \%$ identity in nucleotides to SARS-CoV, the seven conserved replicase domains in ORF1ab has $94.6 \%$ sequence identity in amino acids between SARS-CoV-2 and SARS-CoV [1], suggesting the possibility that these two viruses might belong to the same species.

Genome sequencing showed that the genome organization of all bat SARS-like-CoVs is almost identical to that of the SARS-CoVs iso- 
lated from humans or civets, and they shared an overall sequence identity of $88 \%$ to $92 \%[2,3]$. The sequence identity between SARS-CoV-2 and bat-SL-CoVZC45 or bat-SL-CoVZXC21, the closest relatives, is lower than $90 \%$ [2]. However, SARS-CoV-2 has $96.2 \%$ overall genome sequence identity to Bat-CoV-RaTG13, a bat coronavirus detected in Rhinolophus affinis from Yunnan province [1]. Bats are thus considered to be the potential natural host of SARScoronaviruses.

Palm civets were implicated to be the intermediate host for SARS-CoV. The genome sequences of SARS-CoVs from palm civets were almost identical to the genomes from human SARS-CoVs $[3,4]$. The intermediate host for SARS-CoV-2 is suggested to be the pangolin as the genome similarity between SARS-CoV-2 and the identified coronaviruses from pangolins is approximately $85.5 \%$ to $92.4 \%$, lower than that seen with the Bat-CoV-RaTG13 [1,5]. Interestingly, the pangolin coronavirus and SARS-CoV-2 share identical amino acids at the five critical residues of RBD of S protein, while the bat coronavirus RaTG13 shares such identity with SARSCoV-2 only in one residue. However, the receptor-binding domain of $\mathrm{S}$ protein from one sub-lineage of the pangolin coronaviruses shows $97.4 \%$ similarity in amino acid sequences to that of SARSCoV-2, even higher than that seen with Bat-CoV-RaTG13 (89.2\%) [5]. Although other possible intermediate hosts for SARS-CoV-2 including snakes, raccoons, cats, mice have also been suggested, no conclusive evidence has been found.

Foodborne spread and/or close contact is thought to be the mode of zoonotic transmission from the primary reservoir or the intermediate host to human beings, however, the exact route has not been elucidated, and the precise mechanism of this cross-species leap still remains a mystery.

\section{Microbiology}

The SARS-coronaviruses belong to the genus Betacoronavirus, subgenus Sarbecovirus and subgroup $2 B$. They are are large, spherical to pleomorphic particles. The diameter of virus particles of SARS-CoV-2 and SARS-CoV, ranges from 60 - $140 \mathrm{~nm}$ [6] and 100 - $130 \mathrm{~nm}$ [7], respectively. The virion particles are composed of an outer envelope, and the genomic RNA enclosed within helical nucleocapsid (Figure 1).

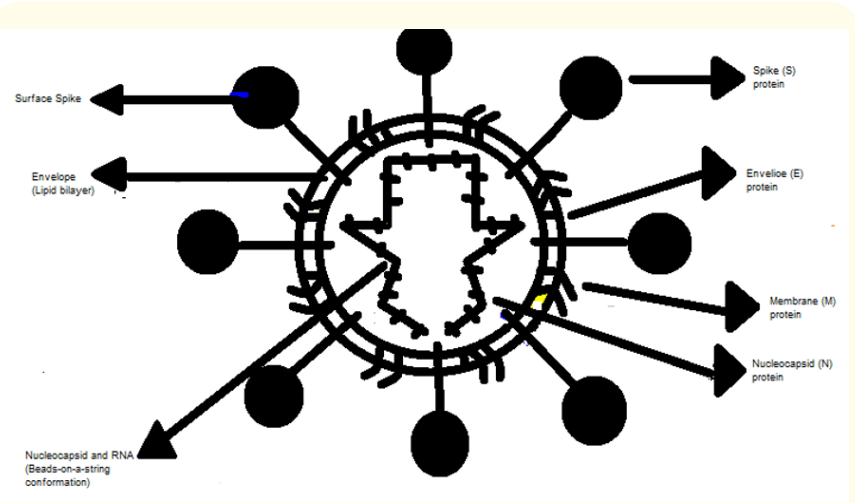

Figure 1: Structural organization of coronavirus.

Envelope

The envelope consists of a lipid bilayer in which the Envelope (E), Spike (S) and Membrane (M) structural proteins are embedded and anchored [8]. The ratio of E:S:M in the lipid bilayer is approx. 1:20:300 [9]. The envelope of the virus in electron micrographs appears as a distinct pair of electron-dense shells which are relatively opaque to the electron beam used to scan the virus particles $[10,11]$.

\section{Envelope (E) protein}

Envelope (E) protein is a small ( $\sim 8-12 \mathrm{kDa} ; \sim 76-109$ amino acids), hydrophobic, integral protein present in the lipid bilayer. Composed of an N-terminal domain, a long alpha-helical transmembrane domain, and a C-terminal hydrophilic domain, this protein is proposed to have multiple membrane topologies. E protein is involved in critical aspects of the virus life cycle such as assembly, envelope formation, and viral egress (by altering host secretory pathway) as well as maintains the structural shape of the virus particle. It functions as an ion-channeling viroporin and interacts with other coronavirus proteins and host cell proteins. Although the exact mechanism remains unclear, this protein also has a role in the host stress response. The coronaviruses lacking E protein make promising vaccine candidates $[12,13]$.

\section{Spike (S) protein}

The spike (S) protein is a transmembrane glycoprotein that assembles into homotrimers (oligomers) on the virion surface to form the spikes. It is a large $(\sim 180-200 \mathrm{kDa})$, class I fusion protein 
that has the characteristic alpha-helical coiled-coil structure. It is composed of 1100 - 1600 amino acids and is highly glycosylated as it contains 21 - $35 \mathrm{~N}$-glycosylation sites [15]. The function of S protein is to mediate receptor binding and membrane fusion between the virus and the host cell. Moreover, $\mathrm{S}$ protein is the common target of neutralizing antibodies and thus, it is the most commonly used viral antigen in the production of vaccines [15-19].

The surface spike has 3 segments: (a) Ectodomain, (b) Singlepass transmembrane anchor domain, and (c) Short intracellular tail [14]. The ectodomain of S protein (Figure 2) is composed of 2 subunits- S1 and S2. S1 subunit (N-terminal), the globular head of the spike, contains 2 subdomains- an N-terminal domain (NTD) and a C-terminal domain (CTD). The C-terminal domain serves as the receptor binding domain (RBD) and binds proteinaceous receptors exclusively. S2 subunit (C-terminal) forms the stem which anchors the spike in the viral envelope, and contains 2 Heptad repeats (HRs), a typical feature of class I viral fusion proteins. The heptad repeats comprise a repetitive heptapeptide, abcdefg, with a and $d$ being hydrophobic residues, characteristic of the formation of coiled-coil that participates in the fusion process [16-19].

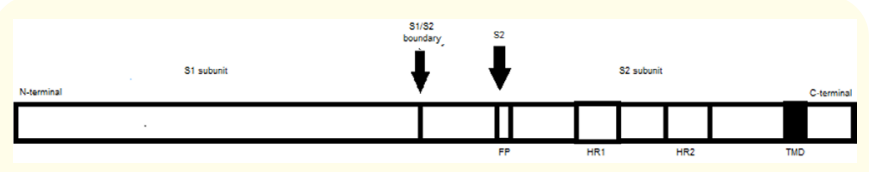

Figure 2: Spike (S) protein of coronavirus.

The given figure depicts the 2 subunits of the spike protein- S1 and $\mathrm{S} 2$. The smaller arrow points to the boundary between S1/S2-the first cleavage site that separates the RBD and Fusion Domain of S protein. The bigger arrow points to the second cleavage site at S2 which harbors the fusion peptide (FP). Following cleavage at S2, the fusion peptide (FP) gets exposed that inserts into the membrane, and the heptad repeats (HR1 and HR2) present in the S2 subunit join to form an antiparallel six-helix bundle (post-fusion conformation). The formation of the bundle allows for the mixing of viral and cellular membranes, resulting in fusion and ultimately, release of the viral genome into the host cell's cytoplasm. TMD represents the transmembrane domain of the spike protein $(\mathrm{S})$.

Some notable differences between the S proteins of SARS-CoV-2 and SARS-CoV have been demonstrated. First, the spike protein of
SARS-CoV-2 is longer than that of SARS-CoV [20]. Second, the S proteins of SARS-CoV-2 share about $76 \%$ of amino acid identities with SARS-CoV, while the amino acid sequence of potential RBD of SARS-CoV-2 is only about $74 \%$ homologous to that of SARS-CoV. Third, there are three short insertions in the N-terminal domain of SARS-CoV-2 which may confer to sialic acid binding activity, and four out of five key residue changes in the receptor-binding motif, as compared to SARS-CoV. Fourth, the S glycoprotein of SARS-CoV-2 harbors a Furin (a Golgi-resident host protease) cleavage site at the boundary between the S1/S2 subunits, which is processed during biogenesis and sets this virus apart from SARS-CoV [21,22].

\section{Membrane (M) protein}

Membrane (M) protein is the most abundant envelope protein. It is a type III transmembrane glycoprotein ( $\sim 230$ amino acids) composed of three parts- a short amino-terminal domain on the outside of the virion membrane, three transmembrane domains, and a long carboxy-terminal domain on the inside of the virion particle. Lateral interactions between $\mathrm{M}$ proteins are thought to mediate the formation of virion envelope and are responsible for maintaining the structural shape of the virion particle. The M protein plays a key role in virus assembly and mediates the incorporation of the spikes into the viral envelope [13].

\section{Nucleocapsid}

Encoded by the $9^{\text {th }}$ ORF of the genome, the nucleocapsid (N) protein is a structural and multifunctional protein, that constitutes the only protein present in the nucleocapsid. It is a $\sim 46 \mathrm{kDa}$ protein composed of 422 amino acids, and has two independent domains, an N-terminal domain (NTD), and a C-terminal domain (CTD). The $\mathrm{N}$-terminal region consists mostly of positively charged amino acids that are responsible for RNA binding. A lysine-rich region present at the C-terminus, is predicted to be capable of self-association. Besides these, an SR (serine arginine)-rich motif is present in the middle region which is highly disordered, plays an important role in effective viral replication, and serves as a linker between the NTD and CTD [23].

$\mathrm{N}$ protein binds the viral genome in a beads-on-a-string type conformation. Two specific RNA substrates have been identified for $\mathrm{N}$ protein to help package the genome- the TRSs (Transcription Regulatory Sequences) and the genomic packaging signal. The $\mathrm{N}$ protein also binds to nsp3, a key component of the replicase-com- 
plex that helps tether the viral genome to the replicase-transcriptase complex, thus enhancing the efficiency of viral transcription. The CTD, and a part of the linker region of N protein, interact with CTD of M protein to aid in the process of virus self-assembly [23].

In the host cell, the $\mathrm{N}$ protein is abundantly produced and causes perturbation of cellular processes that have pathological consequences. The disturbances at the cellular level include- deregulation of host cell cycle, inhibition of host cell cytokinesis, inhibition of host cell translation machinery, inhibition of interferon production, modulation of TGF- $\beta$ signaling pathway, upregulation of COX2 production, upregulation of Activator Protein 1 (AP1) activity, induction of apoptosis, upregulation of prothrombinase gene transcription and association with host cell proteins [23].

The abundance and high hydrophilicity of $\mathrm{N}$ protein contributes to potent immunity after coronavirus infection. SARS-CoV N protein is extremely antigenic as it produces a highly restricted IgGdominated antibody response directed against the nucleocapsid. Thus, N protein can also be used in the production of vaccines [23]. However, SARS-CoV-1 N protein can also inhibit IFN- $\gamma$ response, thus neutralizing the host immune response. This property of $\mathrm{N}$ protein has not yet been determined in SARS-CoV-2, which supports the low mortality caused by this novel coronavirus [24].

\section{Genome}

Coronaviruses contain a linear, positive-sense, single-stranded RNA genome. The genome size is approx. 30 kilobases (kb). Precisely, SARS-CoV-2 has 29,903, and SARS-CoV has 29,751 nucleotides in the complete DNA sequence length [25]. They have one of the largest genomes among RNA viruses. Their genome has a $5^{\prime}$-methylated cap and a $3^{\prime}$-polyadenylated tail, that allows the positive-sense RNA genome to be directly translated by the host cell's ribosome on virus entry. The virus genome has 14 open reading frames (ORFs) which overlap in some cases [11].

The genome organization for a coronavirus is $5^{\prime}$-leader-UTRreplicase/transcriptase-spike (S)-envelope (E)-membrane (M)nucleocapsid (N)-3'UTR-poly (A) tail. The ORFs-1a and 1b, which occupy the first two-thirds of the genome, encode the replicasetranscriptase complex polyprotein (pp1ab). The later ORFs-2, 4, 5 and 9a encode, respectively, the four major structural proteins: spike, envelope, membrane, and nucleocapsid. The later ORFs also encode for eight unique proteins (ORF3a to ORF9b), known as the accessory proteins, the functions of which are not well understood [11].

\section{Life cycle}

\section{Attachment and entry}

The attachment of the virus to the host cell is mediated by the interaction between spike protein on the surface of the virion particle, and its corresponding receptor within the host tissues. In humans, the RBD at the C-terminus of the S1 subunit recognizes and attaches to the Angiotensin-converting enzyme 2 (ACE2) receptor [26]. This interaction is the primary determinant for coronavirus to infect a host species and also governs the tissue tropism of the virus. Following receptor binding, the virus can gain access to the host cell cytosol by different paths, depending upon the host protease available to cleave, and activate the receptor-attached spike protein [27].

The virus entry into the cell cytosol is generally accomplished by acid-dependent proteolytic cleavage of the S protein by the serine protease, TMPRSS2, or TMPRSS11D, on the cell surface [28]. The activation of the $\mathrm{C}$-terminal part of the spike protein triggers the fusion of the viral envelope with the host cell's membrane by inducing conformational changes [29]. This mechanism is less likely to trigger host cell antiviral immunity, and is, therefore, more efficient for viral replication. Alternatively, the virus can gain access to the host cell cytosol by endocytosis and uptake of the virus in an endosome. The receptor-attached spike protein is then activated by the host's pH-dependent cysteine protease, Cathepsin-L. Activation of the receptor-attached spike protein induces a conformational change, and the subsequent fusion of the viral envelope with the endosomal wall [27]. The precise conformational changes that occur during the fusion of viral and host cellular membranes are not fully understood (Figure 2) [30,31].

\section{Genome translation}

After fusion, nucleocapsid passes into the cytoplasm, where the viral genome is released [11]. The genome acts as a messenger RNA. The host cell's ribosomes translate two-thirds of the genome containing the replicase gene (corresponding to the ORFs-rep1a and rep1b) into two large overlapping co-terminal polyproteins, pp1a and pp1ab. 
To express both the polyproteins, the virus utilizes a slippery sequence (5'-UUUAAAC-3'), and a downstream RNA pseudoknot at the end of ORF1a, which causes ribosomal frameshifting from the ORF rep1a into the ORF rep1b. In most cases, the ribosome unwinds the pseudoknot structure, and continues translation until it encounters the rep1a stop codon. Occasionally, the pseudoknot blocks the ribosome from continuing elongation, causing it to pause on the slippery sequence, changing the reading frame by moving back one nucleotide, a -1 frameshift, before the ribosome can melt the pseudoknot structure, and extend translation into rep1b, resulting in the translation of polyprotein, pp1ab [11].

The polyproteins, pp1a and pp1ab, contain the nonstructural proteins (nsps) 1 - 11 and 1 - 16, respectively. In pp1ab, nsp11 from pp1a becomes nsp12 following extension of pp1a into pp1b. These polyproteins are subsequently cleaved into 16 individual nsps. SARS-coronaviruses encode the following proteases- a single papain-like protease, PLpro (encoded by nsp3) and a serine type protease, 3Clpro or Mpro (encoded by nsp5), that cleave the replicase polyproteins. The PLpro cleaves the nsp1/2, nsp2/3 and nsp3/4 boundaries, while the 3Clpro/Mpro is responsible for the remaining 11 cleavage events [11].

\section{Replication and transcription}

Viral RNA synthesis follows the translation and assembly of the viral replicase complex. Viral RNA synthesis produces both genomic and sub-genomic RNAs. Sub-genomic RNAs serve as mRNAs for the structural, and accessory genes which reside downstream of the replicase polyproteins genes. Because of the relatively large genome size as compared to other RNA viruses, increased fidelity and processivity during RNA synthesis becomes important, which is accomplished with the help of various enzymes; for example, $3^{\prime}$ $5^{\prime}$ Exoribonuclease (ExoN), encoded by nsp14, proofreads the viral genome.

RNA-dependent RNA polymerase (RdRp), encoded by nsp12, is the main replicase-transcriptase complex (RTC) protein that is directly involved in the transcription and replication of RNA, while the other nsps in the complex assist in these processes. During transcription, RdRp directly mediates the synthesis of negative-sense sub-genomic RNA molecules from the positive-sense genomic RNA. This is followed by the transcription of these negative-sense sub-genomic RNA molecules to their corresponding positive-sense mRNAs [11]. During replication of the viral genome,
RdRp directly mediates the synthesis of negative-sense genomic RNA from the positive-sense genomic RNA. This is followed by the replication of positive-sense genomic RNA from the negative-sense genomic RNA [11]. Both genomic and sub-genomic RNAs are produced through negative-strand intermediates that are only about $1 \%$ as abundant as their positive-sense counterparts and contain both polyuridylate and anti-leader sequences [32].

During the coronavirus replication, the leader and body TRS segments fuse during the production of sub-genomic RNAs. It is largely believed to occur during the discontinuous extension of negative-strand RNA. The current model proposes that the RdRp pauses at any one of the body TRS sequences (TRS-B). Following this pause, the RdRp either continues elongation to the next TRS, or it switches to amplifying the leader sequence at the 5 ' end of the genome, guided by the complementarity of the body TRS (TRSB) to the leader TRS (TRS-L). The anti-leader sequence is present at the 3' end of the negative-strand sub-genomic RNAs. However, it is not fully understood how the RdRp bypasses all of the TRS-B sequences to produce full length negative-strand genomic RNA and how the TRS-B sequence gets directed to the TRS-L, and how much complementarity is necessary [11].

The replicated positive-sense genomic RNA becomes the genome of the progeny viruses. The various smaller mRNAs are transcripts from the last third of the virus genome which follow the reading frames, ORF1a and $0 R F 1 b$. These mRNAs are translated into the four structural proteins (S, E, M and N) that become a part of the progeny virus particles, and also eight other accessory proteins which assist the virus in their replication process [11].

\section{Assembly and release}

Following replication and sub-genomic RNA synthesis, the viral structural proteins, S, E, and M are translated and inserted into the endoplasmic reticulum (ER). These proteins move and traffic along the secretory pathway into the endoplasmic reticulum-golgi intermediate component (ERGIC). The viral genomes packaged within the $\mathrm{N}$ proteins, bud into the membrane of ERGIC containing the viral structural proteins, forming the mature virion particles. Once the virion particles are assembled completely, they are transported to the cell surface packaged within vesicles and released by exocytosis [11]. 


\section{Transmission}

The commonest mode of transmission of SARS-CoV-2 and SARS$\mathrm{CoV}$ is 'respiratory droplets' (size $>5$ - 10 microns). These droplets travel from an infected person to a healthy individual while coughing, and/or sneezing, when propelled at a short physical distance, usually up to 3 feet (Direct contact). These droplets released by the infected person come in direct contact with the healthy person's mucous membranes of the nose, mouth and conjunctiva of the eyes, and get deposited over them. The airborne spread occurs through 'droplet nuclei' (size $<5$ microns) that remain infectious when suspended in air over long distances and time, and are produced during coughing, sneezing, breathing, speaking, laughing, and by aerosol-generating procedures such as nebulization, highflow oxygen therapy, endotracheal intubation. Airborne transmission of SARS-CoV-2 and SARS-CoV can occur during aerosol generating procedures $[7,33,34]$. WHO, together with the scientific community, has been actively discussing and evaluating whether SARS-CoV-2 may also spread through aerosols in the absence of aerosol generating procedures, particularly in indoor settings with poor ventilation. Another mode of transmission is through contact with fomites (external surfaces contaminated by the infectious respiratory droplets) (Indirect contact) [33,34].

In recent works, live SARS-CoV-2 has been detected in the stools suggesting the possibility of feco-oral transmission, while SARSCoV-2 RNA has been detected in the urine of a few patients. To date, however, no reports have been published on the transmission of SARS-CoV-2 through either feces or urine [33]. SARS-CoV is also excreted in the stools and feco-oral transmission is strongly suspected [7]. However, such modes of transmission have not been well documented in epidemiological and clinical settings.

\section{The significance of R0 in infectious disease dynamics}

The basic reproductive number $\left(\mathrm{R}_{0}\right)$ of COVID-19 and SARS is, 3.8 [35] and 0.19-1.08 [36] respectively. $A n R_{0}$ value $>1.0$ indicates the infection will be able to start spreading in a population, and it will be harder to control the epidemic. The different characteristics of the COVID-19 pandemic compared to the 2003 SARS outbreak can be hinted by its relatively higher $\mathrm{R}_{0}$ value, that is associated with longer incubation period, mild-to-moderate symptoms or latent infection, and a low mortality rate [37].
Pathogenesis

Pulmonary damage

When the respiratory droplet containing the virus enters the nose, nasopharynx, or oropharynx, it infects the lining epithelial cells. Local replication of the virus stimulates innate immune response which is the first line of defense against foreign invaders. The virus burden at this stage is low, but the host becomes infectious. The infection further propagates and the virus migrates further down from the upper airways to the lower respiratory tract. The immune system elicits a stronger response, and adaptive immunity comes into action. Virus-infected epithelial cells produce IFN- $\beta$, and IFN $-\lambda$ to protect the adjacent cells from invasion by the virus particles. SARS-coronaviruses infect type 2 pneumocytes preferentially. The virus replicates within these cells to produce a large army of progeny virion particles which get released to infect the adjacent cells. The infected host cells undergo apoptosis due to the cytopathic effects of the virus. ACE2 is highly expressed on the apical surface of the alveolar epithelial cells. The changes in the levels of angiotensin-converting enzyme 2 and related molecules leads to increased vascular permeability, along with destruction of alveolar lining epithelium. This leads to acute lung injury, characterized by diffuse alveolar damage (DAD) with the formation of hyaline-rich fibrous membranes, and a few multinucleated giant cells. The virus spills into the peripheral blood from the lungs. Increased expression of ACE2 receptors on the endothelial lining of the blood vessels increases the risk for endothelial damage, and thus, thrombotic complications [38].

\section{Immune response}

Alveolar epithelial cells, pulmonary alveolar macrophages (PAMs), and dendritic cells (DCs) are the immune cells responsible for innate immunity in the airway. PAMs are located on the apical surface of alveolar epithelial cells, and DCs are present underneath the alveolar epithelium. ACE2 receptors are highly expressed on the apical side of alveolar epithelium while the evidence for the presence of ACE2 receptors on macrophages, and DCs is limited. It has been observed that SARS-CoV binds to dendritic cell-specific intercellular-adhesion-molecule-3-grabbing nonintegrin (DCSIGN), and DC-SIGN related proteins (DC-SIGNR, L-SIGN) that are highly expressed on DCs and macrophages. However, the presence of such additional receptors to which SARS-CoV-2 binds needs fur- 
ther research. Infection of immune cells by SARS-coronaviruses is usually abortive, though the production of cytokines, and chemokines continue [38].

PAMs and DCs phagocytose the virus-infected apoptotic cells and move to the nearby draining lymph nodes for antigen presentation to T cells. CD4+ cells activate B cells to stimulate the production of virus-specific antibodies (polyclonal response), while CD8+ $\mathrm{T}$ cells are recruited to kill the virus-infected cells. Lymphopenia has been observed in these patients. There is a decrease in both CD4+ and CD8+ cells, however, their ratio remains unaffected [39]. Direct infection of $\mathrm{T}$ cells by SARS-coronaviruses has been proposed as one of the explanations for this finding. It may also be caused by cortisol production from activation of the hypothalamicpituitary-adrenal axis in response to stress. However, the precise mechanism is not well understood.

There is an upregulation of acute-phase reactants like serum amyloid A, and mannose-binding lectin (MBL). MBL has been shown to lower the infectivity of the virus by binding and blocking the carbohydrate-recognition domain of S protein [38]. Elevated levels of C-reactive protein (CRP), a non-specific inflammatory marker, have also been observed in these patients, and demonstrate a positive association with the severity of the disease course. Thus, CRP serves as a useful marker in clinical settings to monitor the response to therapy and predict the prognosis.

Cytokine storm in the host is a pathognomonic finding in SARScoronaviruses infections. During the first 2 weeks of illness, the production of proinflammatory cytokines such as IL-1 $\beta$, IL-6, IL-8, IL-12, IFN- $\gamma$, IP-10, and MCP1 by the infected lung epithelial cells, monocytes, macrophages, dendritic cells, is markedly escalated. T cells also increase the secretion of TNF- $\alpha$, IFN- $\gamma$, and IL-2. CXCL10 or IP-10 produced in response to IFN- $\gamma$ signaling, binds to cell surface chemokine receptor, CXCR3 and serves as a chemo-attractant for various cells of the immune system. The levels of CXCL10 can predict the subsequent course of the illness and serve as a useful disease marker. Sometimes, the presence of anti-inflammatory molecules like IL-10, TGF- $\beta$ and PGE $_{2}$ in the serum of SARS patients provides a clue to the prolonged and severe disease course [40]. Inhibition of phagocytosis by macrophages and DCs, is mediated by IL- 6 and IL-8 produced by SARS-coronaviruses infected epithelial cells. Severe inflammation leads to the formation of oxidized phospholipids, which in turn, further stimulates IL-6 production from lung macrophages. IL-8 also serves as a chemoattractant for neutrophils that can cause acute lung injury.

SARS-coronaviruses have evolved various strategies to evade the host antiviral type 1 interferon response, that mediates the switch over from innate immunity to adaptive immunity, to prolong viral replication and survival. Patients with poor outcomes have demonstrated dysregulated type 1 and type 2 interferon responses [41].

\section{Clinical presentation}

The incubation period for COVID-19 is thought to extend to 14 days, with a median time of 4 - 5 days from exposure to symptoms onset [42], while that for SARS is typically 2 to 7 days, although in some cases it may be as long as 10 - 14 days [34]

COVID-19 and SARS, both have a similar clinical presentation with flu-like symptoms such as fever, coryza, dry cough, malaise, and headache, during the initial stages. However, in some patients, the mild illness may progress to severe disease, an atypical viral pneumonia, presenting with high fever or chills, cough with minimal sputum production, and dyspnea (Table 1) [43]. Many cases have been reported with no symptoms including fever (asymptomatic carriers). Critical illness leading to serious complications (Table 1) [44] is more commonly seen in those with comorbid heart disease, and diabetes as well as other pre-existing conditions that weaken the host immunity. Pulmonary thrombosis and embolism leading to respiratory failure can prove to be fatal complications.

\begin{tabular}{|c|c|}
\hline Clinical features & Complications \\
\hline $\begin{array}{ll}\text { - } & \text { Fever }(88.7 \%) \\
\text { - } & \text { Cough }(67.8 \%) \\
\text { - } & \text { Fatigue }(38.1 \%) \\
\text { - } & \text { Sputum production }(33.7 \%) \\
\text { - } & \text { Shortness of breath }(18.7 \%) \\
\text { - } & \text { Sore throat }(13.9 \%) \\
\text { - } & \text { Headache }(13.6 \%) \\
\text { - } & \text { Diarrhea }(3.8 \%) \\
\text { - } & \text { Vomiting }(5.0 \%) \\
\text { - } & \text { New loss of taste or smell }\end{array}$ & $\begin{array}{l}\text { - } \text { Sepsis } \\
\text { - } \text { Respiratory failure } \\
\text { - } \text { Acute respiratory dis- } \\
\text { tress syndrome (ARDS) } \\
\text { - } \text { Heart failure } \\
\text { - Septic shock } \\
\text { - } \text { Coagulation dysfunction } \\
\text { - } \quad \text { Liver failure (rare) }\end{array}$ \\
\hline
\end{tabular}

Table 1: Clinical presentation of COVID-19. 


\section{Diagnosis}

The diagnosis of COVID-19 and SARS can be made using the following modalities- RT-PCR detection of viral RNA in clinical samples, serologic detection of specific antibodies to the virus, and growth of the virus in cell culture.

For all practical purposes, real-time RT-PCR, a type of nucleic acid amplification test (NAAT), is used for the diagnosis of these illnesses, considering the turnaround time and ease of administration. This test can be carried out on various types of samples such as upper respiratory specimens (preferably nasopharyngeal swab, or oropharyngeal swab), and/or lower respiratory specimens (sputum, if produced, endotracheal aspirate or bronchoalveolar lavage) in patients with severe respiratory disease, blood sample, or stool sample and/or rectal swab. The suspected cases of COVID-19 are confirmed by detection of unique sequences of virus RNA using RT-PCR with additional confirmation by nucleic acid sequencing when necessary. The viral genes targeted for detection include the $\mathrm{N}, \mathrm{E}, \mathrm{S}$, and RdRp genes. RNA extraction should be done in a laboratory with biosafety level II facility [45].

Serologic testing to detect virus-specific IgM and IgG antibodies in serum samples can also be done using IFA (Immunofluorescence Assay) or ELISA (Enzyme-linked Immunosorbent Assay). Such serologic assays can aid investigation of an ongoing outbreak and retrospective assessment of the attack rate or extent of an outbreak. In cases where NAAT assays are negative and there is a strong epidemiological link to COVID-19 infection, paired serum samples (in the acute and convalescent phase) might support diagnosis once validated serology tests are available. Serum samples can be stored for these purposes [45]. Cross reactivity to other coronaviruses can be challenging [46] and confirmation by virus neutralization must be considered to avoid the possibility of false positives due to other human coronaviruses-OC43 and 229E.

Isolation of SARS-coronavirus using viral culture from any specimen would be confined to a laboratory with biosafety level III containment and should also be confirmed second time by nucleic acid sequencing when necessary. This method is not routinely employed for clinical diagnosis due to the risk of spreading the virus, and is mainly reserved for research activities. Human airway epithelial cell lines were used for the isolation of the virus initially [47].

\section{Therapeutic interventions}

The management protocols for COVID-19 and SARS focus mainly on supportive care to provide symptomatic relief. Nonetheless, several targeted chemotherapeutic agents have been used in clinical settings and have shown positive results (Table 2) [48-50]. The recent advances in the treatment of COVID-19 are briefly discussed below.

Antivirals
Protease inhibitors: Lopinavir, Ritonavir
Nucleoside analogs: Ribavirin
Immunomodulators
Corticosteroids
Interferons: IFN- $\alpha$, IFN- $\beta$
Intravenous Immunoglobulins (IVIG)
Other agents
Zinc
IL-6 receptor blocker: Tocilizumab
Recent advances
Remdesivir
Favipiravir
Chloroquine
Ivermectin

Table 2: Chemotherapeutic agents for the treatment of COVID-19 and SARS.

Remdesivir is an adenosine analog with broad-spectrum antiviral activity. It gets incorporated into the viral RNA and causes premature chain termination. Favipiravir, a guanine analog, was first developed for the treatment of avian influenza. It targets conservative catalytic domain of RNA-dependent RNA polymerase, interrupting the addition of nucleotides during viral RNA replication. Recent in vitro studies have shown Favipiravir to be safe and effective against SARS-CoV-2 [49].

Chloroquine is an antimalarial drug which has proven efficacy as an antiviral agent against SARS-coronaviruses. It increases endosomal $\mathrm{pH}$, and thus makes the environment unfavorable for the virus/host cell fusion. It also prevents further spread of the SARScoronaviruses. Chloroquine affects the glycosylation of angioten- 
sin-converting enzyme-2 (ACE2) receptor, an essential process for binding the viral spike protein. Moreover, it has immunomodulatory effects such as suppressing the production, and release of TNF- $\alpha$ and IL-6. It also works as a novel class of autophagy inhibitor, which may interfere with viral infection and replication. A combination of Remdesivir and Chloroquine has shown to effectively inhibit SARS-CoV-2 during in vitro experiments [49].

Ivermectin, an anti-parasitic agent, has demonstrated antiviral properties in vitro. The antiviral activity is mediated by inhibition of Importin (IMP) $\alpha / \beta$ receptor, which is responsible for transporting viral proteins into the host cell nucleus, an essential part of the virus life cycle. This property of ivermectin is being tested in vivo as a part of various clinical trials [50].

\section{Conclusion}

Despite being identical, SARS-CoV-2 and SARS-CoV have caused two different kinds of pandemics. SARS-CoV-2 has proven to be more infectious and transmissible while SARS-CoV was more virulent. Since SARS-CoV-2 has created havoc that is difficult to control, further research studies are required to answer a few questions. It is important to identify the stages at which the chain of transmission can be interrupted. The role of community transmission needs to be investigated. It remains unknown whether antibodies from the previous infection will offer short-term or long-term protection, if the SARS-CoV-2 mutates over time.

\section{Conflicts of Interest and Source of Funding}

None declared.

\section{Bibliography}

1. Zhou P., et al. "A Pneumonia Outbreak Associated with a New Coronavirus of Probable Bat Origin". Nature 579.7798 (2020): 270-273.

2. Guan Y., et al. "Isolation and Characterization of Viruses Related to the SARS Coronavirus From Animals in Southern China". Science 302.5643 (2003): 276-278.

3. Song HD Tu CC., et al. "Cross-host evolution of severe acute respiratory syndrome coronavirus in palm civet and human". Proceedings of the National Academy of Sciences of the United States of America 102.7 (2005): 2430-2435.
4. Chinese SARS Molecular Epidemiology Consortium. "Molecular Evolution of the SARS Coronavirus During the Course of the SARS Epidemic in China". Science 303.5664 (2004): 16661669.

5. Lam TT., et al. "Identification of 2019-nCoV related coronaviruses in Malayan pangolins in southern China". Nature 583 (2020): 282-285.

6. Marco Cascella., et al. "Features, Evaluation and Treatment Coronavirus (COVID-19). Stat Pearls Internet (2020).

7. AABB. Transfusion Medicine. "Emerging Infectious Disease Agents". Transfusion 49 (2009): 150S-152S.

8. Lai MM and Cavanagh D. "The molecular biology of coronaviruses". Advances in Virus Research 48 (1997): 1-100.

9. Cavanagh D., et al. "Detection of a Coronavirus from Turkey Poults in Europe Genetically Related to Infectious Bronchitis Virus of Chickens". Avian Pathology 4 (2001): 355-368.

10. Neuman BW., et al. "Supramolecular Architecture of Severe Acute Respiratory Syndrome Coronavirus Revealed by Electron Cryomicroscopy". Virology Journal 80.16 (2006): 79187928.

11. Fehr AR and Perlman S. "Coronaviruses: An Overview of Their Replication and Pathogenesis". Methods in Molecular Biology1282 (2015): 1-23.

12. Schoeman D and Fielding BC. "Coronavirus envelope protein: current knowledge". Virology Journal 16.1 (2019): 69.

13. Alsaadi EA and Jones IM. "Membrane binding proteins of coronaviruses". Future Virology 14.4 (2019): 275-286.

14. Li F. "Structure Function, and Evolution of Coronavirus Spike Proteins". Annual Review of Virology 3.1 (2016): 237-261.

15. Hulswit RJ., et al. "Coronavirus Spike Protein and Tropism Changes". Advances in Virus Research 96 (2016): 29-57.

16. Belouzard S., et al. "Mechanisms of Coronavirus Cell Entry Mediated by the Viral Spike Protein". Viruses 4.6 (2012): 10111033. 
17. Heald-Sargent $\mathrm{T}$ and Gallagher T. "Ready, Set, Fuse! The Coronavirus Spike Protein and Acquisition of Fusion Competence". Viruses 4.4 (2012): 557-580.

18. Du L., et al. "The Spike Protein of SARS-CoV- a Target for Vaccine and Therapeutic Development". Nature Reviews Microbiology 7.3 (2009): 226-236.

19. Xu Y., et al. “Crystal Structure of Severe Acute Respiratory Syndrome Coronavirus Spike Protein Fusion Core". Journal of Biological Chemistry 279 (2004): 49414-49419.

20. Lu R., et al. "Genomic Characterisation and Epidemiology of 2019 Novel Coronavirus: Implications for Virus Origins and Receptor Binding". Lancet 395.10224 (2020): 565-574.

21. Walls AC., et al. "Structure, Function, and Antigenicity of the SARS-CoV-2 Spike Glycoprotein". Cell 181.2 (2020): 281-292.

22. Wan Y., et al. "Receptor Recognition by the Novel Coronavirus from Wuhan: An Analysis Based on Decade-Long Structural Studies of SARS Coronavirus". Journal of Virology 94.7 (2020): e00127-e00120.

23. Surjit M and Lal SK. "The SARS-CoV nucleocapsid protein: A protein with multifarious activities". Infection, Genetics and Evolution 8.4 (2008): 397-405.

24. Li G., et al. "Coronavirus Infections and Immune Responses". Journal of Medical Virology 92.4 (2020): 424-432.

25. Xu J., et al. "Systematic Comparison of Two Animal-to-Human Transmitted Human Coronaviruses: SARS-CoV-2 and SARSCoV". Viruses 12.2 (2020): 244.

26. Gheblawi M., et al. "Angiotensin-Converting Enzyme 2: SARSCoV-2 Receptor and Regulator of the Renin-Angiotensin System". Circulation Research 126.10 (2020).

27. Simmons G., et al. "Proteolytic Activation of the SARS-coronavirus Spike Protein: Cutting Enzymes at the Cutting Edge of Antiviral Research". Antiviral Research 100.3 (2013): 605-614.

28. Heurich A., et al. "TMPRSS2 and ADAM17 Cleave ACE2 Differentially and Only Proteolysis by TMPRSS2 Augments Entry Driven by the Severe Acute Respiratory Syndrome Coronavirus Spike Protein". Journal of Virology 88.2 (2014): 1293-1307.
29. Li Z., et al. "The human coronavirus HCoV-229E S-protein structure and receptor binding". eLife 8 (2019): e51230.

30. Belouzard S., et al. "Activation of the SARS Coronavirus Spike Protein via Sequential Proteolytic Cleavage at Two Distinct Sites". Proceedings of the National Academy of Sciences of the United States of America 106.14 (2009): 5871-5876.

31. Bosch BJ., et al. "The Coronavirus Spike Protein Is a Class I Virus Fusion Protein: Structural and Functional Characterization of the Fusion Core Complex". Journal of Virology 77.16 (2003): 8801-8811.

32. Sethna PB., et al. "Minus-strand Copies of Replicating Coronavirus mRNAs Contain Antileaders". Journal of Virology 65.1 (1991): 320-325.

33. World Health Organization (WHO). "Transmission of SARSCoV-2: implications for infection prevention precautions (2020).

34. Centers for Disease Control and Prevention (CDC). Severe Acute Respiratory Syndrome (SADS). Frequently Asked Questions About SARS (2020).

35. Australian Government, Department of Health. Novel coronavirus (COVID-19). Information for Clinicians: Frequently Asked Questions.

36. Chowell G., et al. "Model Parameters and Outbreak Control for SARS”. Emerging Infectious Diseases 10.7 (2004): 1258-1263.

37. Chen Y., et al. "Emerging coronaviruses: genome structure, replication and pathogenesis". Journal of Medical Virology 92.4 (2020): 418-423.

38. Gu J and Korteweg C. "Pathology and Pathogenesis of Severe Acute Respiratory Syndrome". The American Journal of Pathology 170.4 (2007): 1136-1147.

39. Wong RS., et al. "Haematological Manifestations in Patients With Severe Acute Respiratory Syndrome: Retrospective Analysis". British Medical Journal 326.7403 (2003): 1358-1362.

40. Huang KJ., et al. "An Interferon-Gamma-Related Cytokine Storm in SARS Patients". Journal of Medical Virology 75.2 (2005): 185-194. 
41. Cameron MJ., et al. "Interferon-mediated Immunopathological Events Are Associated With Atypical Innate and Adaptive Immune Responses in Patients With Severe Acute Respiratory Syndrome". Journal of Virology 81.16 (2007): 8692-8706.

42. Centers for Disease Control and Prevention (CDC). Coronavirus Disease 2019 (COVID-19). Interim Clinical Guidance for Management of Patients with Confirmed Coronavirus Disease (COVID-19).

43. Guan WJ., et al. "Clinical characteristics of coronavirus disease 2019 in China". The New England Journal of Medicine 382 (2020): 1708-1720.

44. Zhou F., et al. "Clinical course and risk factors for mortality of adult inpatients with COVID-19 in Wuhan, China: a retrospective cohort study". Lancet 395.10229 (2020): 1054-1062.

45. WHO. Laboratory testing for 2019 novel coronavirus (2019$\mathrm{nCoV}$ ) in suspected human cases (2020e).

46. Meyer B., et al. "Serological Assays for Emerging Coronaviruses: Challenges and Pitfalls". Virus Research 194 (2014): 175-183.

47. Zhu N., et al. "A Novel Coronavirus From Patients With Pneumonia in China, 2019". The New England Journal of Medicine 382.8 (2020): 727-733.

48. Zumla A., et al. "Reducing Mortality From 2019-nCoV: HostDirected Therapies Should Be an Option". Lancet 395.10224 (2020): e35-e36.

49. Wang M., et al. "Remdesivir and Chloroquine Effectively Inhibit the Recently Emerged Novel Coronavirus (2019-nCoV) In Vitro". Cell Research 30.3 (2020): 269-271.

50. Heidary F and Gharebaghi R. "Ivermectin: A Systematic Review From Antiviral Effects to COVID-19 Complementary Regimen". The Journal of Antibiotics (2020).

\section{Assets from publication with us}

- Prompt Acknowledgement after receiving the article

- Thorough Double blinded peer review

- Rapid Publication

- Issue of Publication Certificate

- High visibility of your Published work

Website: www.actascientific.com/

Submit Article: www.actascientific.com/submission.php

Email us: editor@actascientific.com

Contact us: +919182824667 